\title{
Therapiestrategien im Alltag (Steroide, Antihistaminika)
}

Ziel einer jeden therapeutischen Intervention ist die kausale Behandlung des Krankheitsprozesses sowie ggfs. ein symptomatischer Ansatz zur Linderung der akuten Beschwerden. Für den Bereich der allergischen Erkrankungen mit den zum Teil unspezifischen Symptomen setzt dies in besonderem Maße eine qualifizierte Anamnese und Diagnostik voraus. Die tägliche Praxis zeigt nicht selten sogar einen eher unkritischen Umgang mit der Diagnose „Allergie“ und dem Einsatz antiallergischer Medikation. Auch findet in einem nicht unerheblichen Maß Selbst- und Laienmedikation statt, was zudem wiederum den Zugang zu allergologischer Diagnostik und Therapie erschwert.

Juckreiz stellt vor allem aus Patientensicht ein wesentliches Symptom allergischer Erkrankungen dar, führt zu erheblichen Einschränkungen der Produktivität und Konzentrationsfähigkeit sowie zu Schlafstörungen und damit insgesamt zu deutlichen Beeinträchtigungen der Lebensqualität [1].

Ebenfalls starke Beeinträchtigungen der o. a. Lebensbereiche gehen von rhinokonjunktivalen und/oder asthmatischen Beschwerden und nicht zuletzt auch von (sichtbaren) Hautausschlägen sowie Hautschwellungen, vor allem im Kopf-/Hals-Bereich, aus. Gerade Angioödeme stellen für die Patienten sehr bedrohliche Ereignisse dar und gehen deshalb einher mit deutlich reduzierter Lebensqualität und Angststörungen vielfältiger Ausprägung.

Demzufolge stellen Antihistaminika (AH) eine Gruppe von Arzneimitteln dar, denen sowohl in der Selbstmedikation als auch in der täglichen Praxis der Behandlung allergischer Erkrankungen eine hohe Relevanz zukommt [2]. Ihre Wirkung besteht in der kompetitiven Blockade des H1-(Histamin-)Rezeptors. Sie stehen für die topische (Augentropfen, Nasensprays) und die systemische Applikation (Tropfen, Säfte, Tabletten und Injektionslösungen) zur Verfügung. Wegen der Vielzahl der Wirkstoffe und Präparate mit unterschiedlichen Labels (derzeit sind über 50 Präparate im Handel) tut eine klare Systematik Not, denn vielfach stimmen Indikation,
Dosierung sowie Applikationszeiträume und -intervalle nicht überein. Zusätzlich sind die OTC- (OTC = over the counter = nicht verschreibungspflichtig) und Arzneimittelrichtlinien (AM-RL) [3] zu beachten. Die Beachtung dieser Vorgaben ist aber nicht nur für den therapeutischen Nutzen und die Sicherheit des Patienten wichtig, sondern auch für die Verordnungssicherheit des Vertragsarztes, denn Prüfanträge wegen „Sonstigen Schäden“ durch $\mathrm{AH}$ Verordnungen stellen bundesweit ein großes Problem dar. In zahlreichen Leitlinien [4] zu den einzelnen Krankheitsbildern wird insbesondere darauf verwiesen, nur noch Substanzen der 2. Generation, also $\mathrm{AH}$ mit geringem bis nicht-sedierendem Potenzial einzusetzen.

Glukokortikosteroide (CS) stellen für zahlreiche Erkrankungen oftmals die einzige Therapieoption dar. In der Allergologie sind sie ebenfalls von grundsätzlicher antiinflamatorischer Bedeutung, weil sie durch intrazelluläre Bindung an GlukokortikoidRezeptoren allergisch ausgelöste Entzündungsprozesse hemmen. Damit stellen topische Glukokortikosteroide (TCS) eine Basismedikation bei allergischem Asthma bronchiale (Dosieraerosole und Pulverinhalatoren) und (mittelschwerer bis schwerer) Rhinokonjunktivitis (Nasensprays und Augentropfen) dar. TCS oder gar Kombinationen aus $\mathrm{AH}$ und TCS aus dem OTC-Bereich haben in der Allergologie außerdem bei der Laien-Behandlung von Bagatellen (einfache Insektenstichreaktionen) und bei den allergischen Kontaktekzeme einen Stellenwert. Allerdings sollten Behandlungsversuche einer Urtikaria mit TCS, oft Triamcinolon- oder Betamethason-Präparaten, endlich der Vergangenheit angehören. Sicherlich handelt es sich dabei aber auch in erster Linie um ein Problem der richtigen Diagnosestellung.

Systemische Glukokortikosteroide (orale oder parenterale Applikation) sollten im Regelfall der Notfall-Intervention vorbehalten sein und auf keinen Fall als Daueroder Langzeit-Therapeutikum eingesetzt werden. In der Realität werden wir aber immer noch mit anderen Praktiken konfrontiert, so nicht selten der „3-Monats-Allergiespritze“ bei Rhinokonjunktivitis, die sich dann als Volon A40 Depot-Injektion entlarven lässt. Zum indikationsgerechten Einsatz in der Praxis sollte die schnelle Verfügbarkeit im Praxisteam trainiert sein. Für die patienteneigene Notfallmedikation muss ebenfalls die schnelle und einfache Verfügbarkeit gewährleistet sein; so macht es z.B. keinen Sinn, einem Nahrungsmittel- oder Insektengiftallergiker ein OCS zu verordnen, das in der entsprechenden Anaphylaxiesituation dann als Tablette geschluckt werden muss.

Bei der oben schon kurz angesprochenen Urtikaria handelt es sich im Regelfall nicht um eine allergische Erkrankung im eigentlichen Sinne, deshalb findet sich diese Erkrankung auch nicht in der Agenda dieses Symposiums (obwohl die Atopische Dermatitis wiederum vertreten ist). Da die Behandlung der akuten und besonders der chronischen Urtikaria-Formen aber ein kompliziertes strategisches Zusammenspiel mit dem Wirkstoff Omalizumab (OMA) und den bereits aufgeführten $\mathrm{AH}$ und OCS erfordert, und wir in der tägliche Praxis hier ein für den Patienten schwer zu verstehendes Paradebeispiel zwischen divergierender Leitlinien-Empfehlung und label-konformer Arzneimitteltherapie haben, erlaube ich mir abschließend dazu hier einige kurze Anmerkungen zum Krankheitsmanagement. Die Behandlung der akuten (spontanen) Urtikaria (asU) findet gemäß AM-RL [3] im Regelfall mit OTC-Präparaten statt, die Dosiseskalation gemäß Leitlinie sollte als Heilversuch wegen der von der Fachinformation abweichenden Empfehlung (teilweise Labelund natürlich Dosierungs-Abweichung) und möglicher Nebenwirkungen dokumentiert werden.

Mit dem Übergang in die chronische spontane Urtikaria (csU) wird es aber richtig komplex, denn auch hier ist das Updosing gemäß Leitlinienempfehlung dem Patienten als medizinisch sinnvoll zu empfehlen. Dieses Updosing ist aber nicht zu Lasten der gesetzlichen Krankenkassen rezeptierbar. Gemäß Leitlinie [5] und Label kann aber auch schon nach Versagen der Einfachdosis eines $\mathrm{AH}$ der 2. Generation auf den IgE-Antikörper Omalizumab übergegangen werden, was in Routine vor dem 
Hintergrund des Wirtschaftlichkeitsgebot (§ 12 SGB V) aber selten umgesetzt wird. Dies bedeutet aber den Balanceakt zwischen der Leitlinien-Empfehlung und für den Patienten kostspieligen Privatverordnung des Updosingbedarfs. In diesem Wirrwarr der verschiedenen Regularien wird außerdem oft übersehen, dass im Falle einer csU (L50.8 G) [6] der Patient sogar den Anspruch auf Verordnung der 1-fachDosis der OTC-AH zu Lasten der gesetzlichen Krankenversicherungen, also auf rotem Rezept, hat. Diese Ausnahmeregelung der AM-RL wird aber oftmals vergessen und nicht umgesetzt.

\section{Interessenkonflikt}

Dr. von Kiedrowski erbringt mit seiner Dienstleistungsfirma $\mathrm{CMS}^{3} \mathrm{GmbH}$ Beratungsleistungen, Registerforschung, Tätigkeiten als Prüfarzt bei interventionellen und nicht-interventionellen Studien, sonstige Dienstleistungen und wissenschaftliche Vorträge für: AbbVie, ALK Scherax, Almirall
Hermal, Amgen. Beiersdorf Dermo Medical, Biofrontera, Biogen, BMS, Boehringer Ingelheim, Celgene, DermaPharm, Foamix, Gilead, Hexal, Janssen-Cilag, Leo, Lilly, Meda, Medac, Menlo, MSD, Novartis, Dr. R. Pfleger, Pfizer, Regeneron, Sanofi, Stallergens, Stiefel GSK, Tigercut und UCB.

\section{Autor}

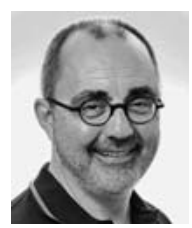

\section{Ralph von Kiedrowski}

Dr. med., Ärztlicher Direktor, PI und Geschäftsführer CMS

Korrespondenzadresse

Dr. med. Ralph von Kiedrowski

$\mathrm{CMS}^{3}$ - Company for Medical Study \& Service Selters $\mathrm{GmbH}$

Kirchstraße 1

56242 Selters

Deutschland

dr.vonkiedrowski@cmss-selters.de

\section{Literatur}

[1] Mlynek A, Magerl M, Hanna M et al. The German version of the Chronic Urticaria Qualityof-Life Questionnaire: factor analysis, validation, and initial clinical findings. Allergy 2009; 64: 927-936

[2] Schwabe U, Paffrath D, Ludwig WD, Klauber J, Hrsg. Arzneimittel-Report 2019

[3] Im Internet (Stand: 27.09.2020): https:// www.g-ba.de/richtlinien/anlage/17/

[4] Im Internet (Stand: 27.09.2020): https:// www.awmf.org/leitlinien/aktuelle-leitlinien/ II-liste/deutsche-dermatologischegesellschaft-ddg.html

[5] Zuberbier T, Aberer W, Asero R et al. The EAACI/GA²LEN/EDF/WAO guideline for the definition, classification, diagnosis and management of urticaria. Allergy 2018; 73 : 1393-1414

[6] Im Internet (Stand: 27.09.2020): https:// www.dimdi.de/static/de/klassifikationen/ icd/icd-10-gm/kode-suche/htmlgm2020/ 